\title{
New challenges and opportunities in adult congenital heart disease
}

\author{
Douglas Moodie
}

In addition to the patients who first present with congenital heart disease as adults, growing numbers of children who were operated on in infancy and young childhood are surviving well into adulthood. In the US, between 800,000 and $1,000,000$ patients over the age of 20 years are estimated to have congenital heart disease-more than among people younger than 20 years. Around 120,000 of these patients have truly complex disease, 300,000 have moderate congenital heart disease, and between 400,000 and 600,000 have simple congenital cardiac defects.

Who is to care for this rising number of adult patients with congenital cardiovascular disease is becoming an increasing problem. A major issue in the care of adult patients with congenital heart disease is the transition that must be made from pediatric to adult care. Pediatric cardiologists rarely see patients older than 18-20 years, especially since it can be difficult for them to do so in the setting of a children's hospital. Some regional centers that have a historical and current interest and expertise in adult congenital heart disease will need to provide most of that care in the US. Most of these centers, however, are in the far west, mid west, or upper northeast, with very few centers in the southeast or southwest.

Another major difficulty is that cardiologists caring for adults are generally poorly trained in congenital heart disease. Undoubtedly, a greater number of pediatric cardiologists need to become acquainted with adult issues in congenital diseases. Very few adult cardiology programs, however, have specific educational opportunities to learn about the care of adult patients with congenital heart conditions; inclusion of every feature of adult cardiology

\section{Who is to care for this rising number of adult patients with congenital cardiovascular disease is becoming an increasing problem}

DS Moodie is the Chairman of the Department of Pediatrics, Ochsner Clinic Foundation, New Orleans, LA, USA.

\section{Competing interests}

The author declared he has no competing interests.

www.nature.com/clinicalpractice doi:10.1038/ncpcardio0275 in the 3-year training program is becoming increasingly difficult. Most adult cardiology programs have no provision for training in congenital heart disease because, although this area is complex and intellectually challenging, the numbers of patients involved are much smaller than those with acquired heart disease. Thus, unless a trainee's interest is stimulated at some point early in his or her fellowship, which usually arises via interaction with pediatric cardiology, individuals seldom seem to acquire or develop a passionate interest in the field, given the little exposure during fellowship.

The sad thing is, the shortage of trained experts coming through the adult cardiology educational programs seems unlikely to change or to improve over the next 5-10 years. Despite two major Bethesda conferences about congenital heart disease in the past 10 years, the number of people with expertise in the field has not grown significantly.

Since the expertise for certain conditions resides with pediatric specialists, exactly who is supposed to provide the care as the patient becomes a young adult is unclear. I think we must be willing to accept at this time that care for adults with congenital heart disease will be limited to the very few institutions that have already demonstrated expertise in postoperative management, complex arrhythmia management, issues of heart failure and transplantation, interventional and electrophysiologic procedures, and detailed long-term follow-up studies; even though these centers are few and not well geographically distributed. This is currently the only way we will prevent adult congenital heart patients from falling through the cracks of our medical system. 